\title{
An Euler System Source Term that Develops Prototype Z-pinch Implosions intended for the Evaluation of Shock-Hydro Methods
}

\author{
J. W. Banks and J. N. Shadid \\ Sandia National Laboratories, \\ Computational Sciences R\&D Group, \\ Electrical and Microsystems Modeling Dept. \\ P.O. Box 5800, Albuquerque NM,87185-1322
}

October 28,2008

\begin{abstract}
In this paper, a phenomenological model for a magnetic drive source term for the momentum and total energy equations of the Euler system is described. This body force term is designed to produce a Z-pinch like implosion that can be used in the development and evaluation of shock-hydrodynamics algorithms that are intended to be used in Z-pinch simulations. The model uses a $\mathbf{J} \times \mathbf{B}$ Lorentz force, motivated by a 0-D analysis of a thin shell (or liner implosion), as a source term in the equations and allows for arbitrary current drives to be simulated. An extension that would include the multi-physics aspects of a proposed combined radiation hydrodynamics (rad-hydro) capability is also discussed. The specific class of prototype problems which are developed is intended to illustrate aspects of liner implosions into a near vacuum and with idealized pre-fill plasma effects. In this work, a high-resolution FCT method implemented on structured overlapping meshes is used to demonstrate the application of such a model to these idealized shock-hydrodynamic studies. The presented results include an asymptotic solution based on a limiting-case thin-shell analytical approximation in both $(x, y)$ and $(r, z)$. Additionally, a set of more realistic implosion problems which include density profiles approximating plasma pre-fill and a set of perturbed liner geometries that excite a hydro-magnetic like Rayleigh-Taylor instability in the implosion dynamics are demonstrated. Finally as a demonstration of including and evaluating multiphysics effects in the Euler system, a simple radiation model is included and self-convergence results for two types of $(r, z)$ implosions are presented.
\end{abstract}

\section{Introduction}

In a typical Z-pinch magnetic implosion, a very large total current $I$ (e.g. $20 \mathrm{MA}$ ) with a characteristic rise time of about 100ns is carried initially by either a thin cylindrical metallic liner or a cylindrical array of metallic wires that ablate and produce a plasma [1]. The current flowing through the conductor/plasma produces a corresponding strong azimuthal magnetic flux, $\mathbf{B}_{\theta}$. The induced local plasma current, $\mathbf{J}$ and magnetic flux density, $\mathbf{B}$, produces a strong $\mathbf{J} \times \mathbf{B}$ Lorentz force which accelerates a highly energetic plasma to stagnate on axis in about 100 ns. This stagnation and subsequent magnetic energy conversion produces an intense $10 \mathrm{~ns} \mathrm{X}$-ray radiation pulse $[2,3]$. The intense $\mathrm{X}$-ray pulse can then be used for radiation-material interaction studies $[4,1,5]$, environments for indirect drive inertial confinement fusion (ICF) applications $[1,5,2]$, or for pursuing laboratory-based astrophysics environments $[5,6,7,8,9,10]$. An important limiting mechanism for the amount of radiation energy produced by a Z-pinch is the Magnetic Raleigh-Taylor (MRT) instability which distorts the outer and inner interfaces of the collapsing plasma shell and broadens the pulse width $[1,5,11]$. A thorough understanding and control of the MRT instability is key to achieving optimal performance of Z-pinch implosions, and for designing the next generation pulsed-power driven Z-pinch machines.

Ideal shock-hydrodynamics (shock-hydro) is a critical foundational technology for simulations of magnetic Z-pinch implosions for computational high energy density physics (HEDP) studies. A minimal Z-pinch 
simulation capability built on this foundation also includes, a multi-material formulation, inclusion of some type of radiation model (rad-hydro), two-temperature physics with plasma thermal conduction phenomena (2T-rad-hydro) and, finally, electromagnetic plasma effects (rad-MHD). This paper presents a phenomenological model for a magnetic drive source term that is designed to help guide development and evaluation of proposed shock-hydro, rad-hydro and finally 2T-rad-hydro simulation capabilities intended for Z-pinch simulations.

One major benefit of this approach is that this evaluation can be carried out for a reasonably realistic set of Z-pinch-like magnetic implosions without requiring a corresponding electro-magnetics capability. For example, this simple source term model can be used in a basic Euler solver for inviscid compressible gases to generate implosions that have the essential character of actual z-pinch implosions. These problems include simple idealized implosions that can be used as verification type problems, for code development purposes, and also for complex implosions with high material velocities, strong-shocks and compressive heating that may generate a radiation power pulse. This paper demonstrates the quantitative use of the proposed $\mathbf{J} \times \mathbf{B}$ source model included in an Euler system solver in two contexts. The first is a verification problem that provides a comparison to an asymptotic analytic 0-D cylindrical solution to the ideal MHD equations. The second type of quantitative study generalizes the Euler system to include a simple radiation-emission term and presents a self-convergence study of the produced radiation pulse for a 1-D cylindrical implosion for the specific shock-hydro algorithm used in this study. This paper also presents results for various forms of cylindrical implosions that contain Rayliegh-Taylor like instabilities and more complex dynamical structures that challenge shock-hydro algorithms. Using these types of problems and various potential extensions, proposed shock-hydro algorithms and solution methods can be verified and evaluated for challenging implosion environments much earlier during algorithmic development without the need for a rad-MHD capability.

In the development that follows, Section 2 briefly states the assumed simplified model of interest which is based on the Euler equations with the possible inclusion of a corresponding radiation model and thermal conduction effects. In Section 3, a classical thin-shell magnetic implosion problem is presented which forms the basis for the development of the phenomenological source term presented in Section 4. This source term is then applied in Section 5 to verification problems based on the thin-shell model and to various prototype magnetic implosion problems. Concluding remarks are then made in Section 7.

\section{General Conservation Law System}

The general format of the conservation law system under consideration is

$$
\frac{\partial \mathbf{U}}{\partial t}+\nabla \bullet \mathbf{F}+\mathbf{S}=\mathbf{0}
$$

In this equation, a general source term $\mathbf{S}$ has been included and will eventually be defined for each individual system of interest. In the following, a few specific forms for such a system are presented.

\subsection{Euler System with 1-Temperature Conduction and Simple Radiation Loss Model}

The specific system of interest is defined generally by

$$
\begin{gathered}
\mathbf{U}=\left[\begin{array}{c}
\rho \\
\rho \mathbf{v} \\
\rho E
\end{array}\right], \quad \mathbf{F}=\left[\begin{array}{c}
\rho \mathbf{v} \\
\rho \mathbf{v} \otimes \mathbf{v}-\mathbf{T} \\
\rho E \mathbf{v}-\mathbf{T} \cdot \mathbf{v}+\mathbf{q}
\end{array}\right], \\
\mathbf{S}=\left[\begin{array}{c}
0 \\
\mathbf{f} \\
\mathbf{f} \cdot \mathbf{v}+Q_{\text {rad }}
\end{array}\right]
\end{gathered}
$$

where the total energy $E$ is defined as the sum of internal energy $e$ and the kinetic energy $\frac{1}{2}\|\mathbf{v}\|^{2}$ as

$$
E=e+\frac{1}{2}\|\mathbf{v}\|^{2} .
$$


The constitutive equations for the material define the stress tensor $\mathbf{T}$ and the heat flux vector $\mathbf{q}$. The source term vector includes the external force per unit volume $\mathbf{f}$, the mechanical work term $\mathbf{f} \cdot \mathbf{v}$, and the radiation energy source term $Q_{\text {rad }}$. The body force term $\mathbf{f}$ will be used in the development of the magnetic drive forcing term of interest for the study in Section 3.

For a thermodynamically simple material, the equation of state (4)-(5) is given by the relations

$$
\begin{aligned}
& P=\hat{P}(\rho e, \rho) \\
& T=\hat{T}(\rho e, \rho)
\end{aligned}
$$

where $P$ is the pressure and $T$ is the temperature. For a specific material, Equations (1)-(2) are closed by the specification of the constitutive relationships for the stress tensor $\mathbf{T}$, the heat flux vector $\mathbf{q}$, and the specific form of the equation of state (4)-(5).

\subsubsection{An Ideal Gas System}

In this section, the system is completed by defining the material dependent constitutive equations and the appropriate equation of state. For an ideal shock-hydrodynamical system

$$
\begin{gathered}
\mathbf{T}=-P \mathbf{I} \\
\mathbf{q}=\mathbf{0}
\end{gathered}
$$

where $\mathbf{T}$ is the stress tensor, and $P$ is the pressure from (4). In the case of a simple ideal gas system, the conduction heat flux is zero and so $\mathbf{q}=\mathbf{0}$. For such an ideal gas the equation of state is given by

$$
P=(\gamma-1) \rho e
$$

where $\gamma$ is the ratio of specific heats. Finally the caloric equation of state for an ideal gas is given by

$$
\rho e=\rho C_{v} T
$$

where $C_{v}$ is the specific heat of the gas at constant volume.

\section{0-D Analysis and the Lorentz Force Term}

In this model, the conducting plasma is considered to be concentrated (or lumped) into a thin shell of inner radius $R$, thickness $\Delta$, and constant initial density $\rho_{L}$. A schematic of the geometry is shown in Figure 1 and the overall system density distribution is depicted graphically in Figure 2. This model also allows for an optional region of plasma pre-fill with density $\rho_{\text {pre-fill }}$ which is considered to be non-conducting, as well as a region of non-conducting foam with density $\rho_{\text {foam }}$. The ability to model a conducting outer shell with inset regions of differing density non-conducting material, allows for developing prototype problems that model aspects of dynamic hohlraums (see e.g. [12, 13, 14]).

Here a lumped mass analysis with the thin shell approximation $\frac{\Delta}{R}<<1$ is considered. Employing these assumptions, the momentum equation in the conservation law is given by Newton's Law of motion for the liner

$$
\mathbf{F}=m \mathbf{a}=\rho_{L} \mathcal{V} \frac{d^{2} R}{d t^{2}} .
$$

Here $\rho_{L}$ and $\mathcal{V}$ are the liner density and the liner volume respectively, $m$ is the mass, $a$ is the acceleration, and $\mathbf{F}$ is the total force given by the Lorentz force term as

$$
\mathbf{F}=(\mathbf{J} \times \mathbf{B}) \mathcal{V} .
$$

In this model the current is confined to the conducting liner material and is oriented in the $z$-direction so that $\mathbf{J}=\left(0,0, J_{z}\right)$. Neglecting the displacement current from Ampère's law then gives 


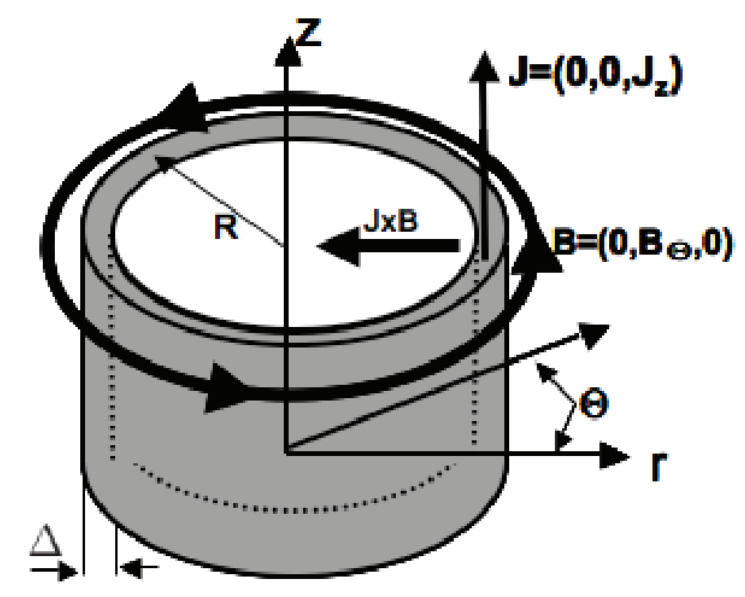

Figure 1: Schematic of prototype thin-shell implosion model.
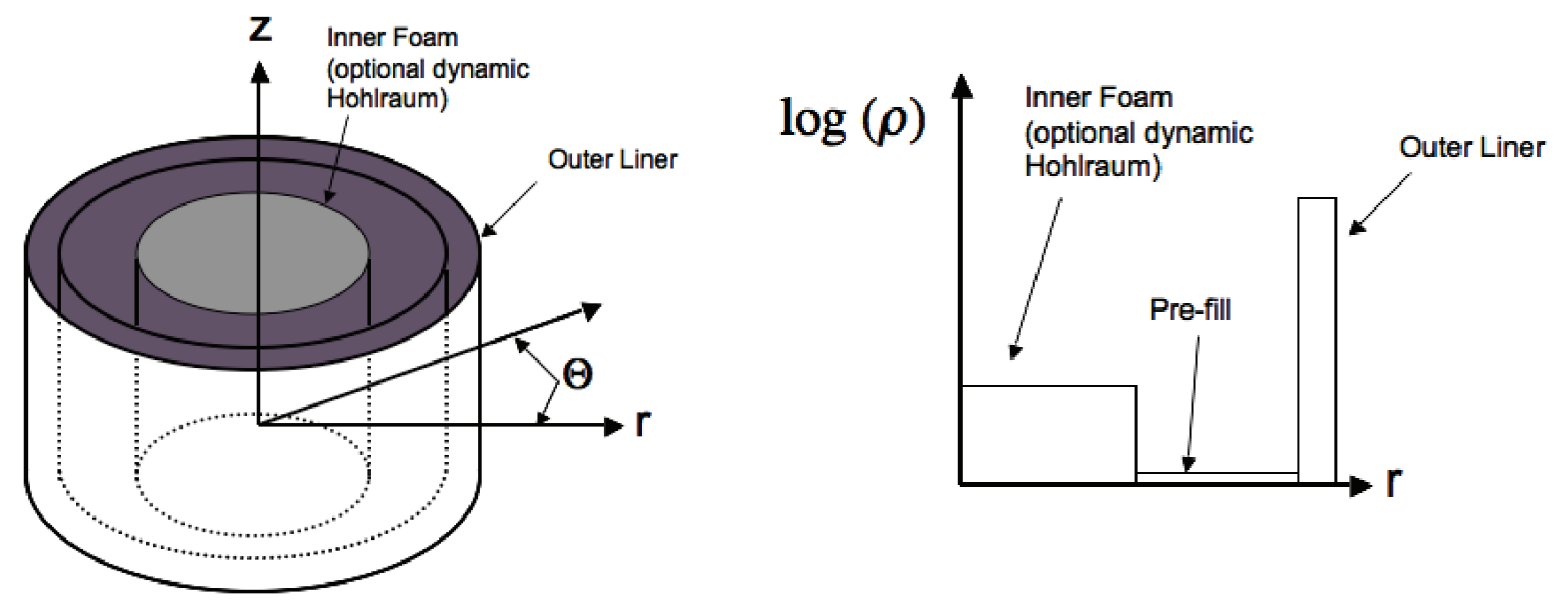

Figure 2: Schematic geometry of the liner with optional pre-fill plasma and inner foam material region for dynamic hohlraum model.

$$
\mathbf{J}=\frac{1}{\mu_{0}} \nabla \times \mathbf{B} .
$$

As a limiting case consider a model in which the liner is assumed to implode in an axisymmetric manner and thus there is assumed to be no variation in $z$ or $\theta$. Therefore Ampère's law reduces to the simpler form

$$
\frac{\partial\left(r B_{\theta}\right)}{\partial r}=r J_{z} \mu_{0}
$$

with flux density $J_{z}=I(t) /(2 \pi R \Delta)$ where $I(t)$ is the total drive current in the liner shell. Integrating and applying the thin shell approximation gives the azimuthal magnetic field

$$
B_{\theta}=\frac{\mu_{0} I(t)}{2 \pi R}
$$

and the total force per unit volume is then given by the Lorentz force term as

$$
\mathbf{f}=-\frac{\mu_{0} I(t)^{2}}{4 \pi^{2} R^{2} \Delta} \hat{\mathbf{e}}_{\mathbf{r}} .
$$


Combination of Equations (10) and (13) with the approximate momentum equation produces the thin shell lumped analysis momentum equation

$$
\rho_{L} \frac{d^{2} R}{d t^{2}}=-\frac{\mu_{0} I(t)^{2}}{4 \pi^{2} R^{2} \Delta}
$$

\subsection{Traditional Thin Shell Analysis}

To recover the more traditional/standard analysis of the thin shell model we consider a per unit axial length analysis of the 0-D model [1]. This analysis takes the mass per unit length, which is often used as a variable by which to calibrate experiments, as a parameter of the system. This analysis is also useful for estimating the approximate energy release for various lengths of cylindrical Z-pinch type implosions. In analyzing the implosion model, the force per unit length is

$$
\mathbf{f}_{l}=-(2 \pi R \Delta) \frac{\mu_{0} I(t)^{2}}{4 \pi^{2} R^{2} \Delta} \hat{\mathbf{e}}_{\mathbf{r}}=-\frac{\mu_{0} I(t)^{2}}{2 \pi R} \hat{\mathbf{e}}_{\mathbf{r}}
$$

where $\beta=\rho_{L}[2 \pi R \Delta]$ is the mass per unit length of the liner. Then the equation of motion on a mass per unit length basis becomes

$$
\beta \frac{d^{2} R}{d t^{2}}=\mathbf{f}_{l} \cdot \hat{\mathbf{e}}_{r}=-\frac{\mu_{0} I(t)^{2}}{2 \pi R} .
$$

To develop a non-dimensional form for the equation of motion (16), the following characteristic scales are employed. The length scale is taken as the initial liner radius $R_{0}$, and the velocity scale as the Alfvèn speed $V_{A}=\frac{\bar{B}}{\left(\bar{\rho} \mu_{0}\right)^{1 / 2}}$, where $\bar{\rho}$ is the average mass density. Here $\bar{\rho}$ is selected as $\bar{\rho}=\beta /\left(2 \pi R_{0}^{2}\right)$ and then using Equation (12) the characteristic time scale becomes the Alfvèn transit time

$$
t_{A}=R_{O} / V_{A}=\sqrt{\frac{2 \pi R_{0}^{2} \beta}{\mu_{0} I_{\max }^{2}}} .
$$

Therefore, the source term in the nondimensional form of the equation of motion is

$$
-\left(\frac{I(t)}{I_{\max }}\right)^{2} \frac{1}{R^{*}} \hat{\mathbf{e}}_{r}
$$

which corresponds to a non-dimensional acceleration, and the non-dimensional thin shell equation of motion (16) becomes

$$
\frac{d^{2} R^{*}}{d t^{* 2}}=-\left(\frac{I(t)}{I_{\max }}\right)^{2} \frac{1}{R^{*}}
$$

To produce the thin shell total energy equation, the Lorentz force per unit length (15) is used and the mechanical work term $\mathbf{f}_{l} \cdot \mathbf{v}$ is computed as

$$
\beta \frac{d E}{d t}=\mathbf{f}_{l} \cdot \mathbf{v} .
$$

The non-dimensional form of the equation is obtained by defining $E^{*}=E /\left(2 \pi V_{A}\right)^{2}$, and of course $V^{*}=$ $V / V_{A}$. With these choices the non-dimensional work can be written as

$$
\left(\frac{I(t)}{I_{\max }}\right)^{2} \frac{V^{*}}{R^{*}}
$$

and the total energy equation becomes

$$
\frac{d E^{*}}{d t^{*}}=\left(\frac{I(t)}{I_{\max }}\right)^{2} \frac{V^{*}}{R^{*}} .
$$


Finally note that using Equation (17) in Equation (19) and integrating it is easily verified that

$$
\hat{E}^{*}=E^{*}(t)-E^{*}(t=0)=\frac{1}{2} V^{* 2}
$$

where it is clear that there is no conversion of kinetic energy to internal energy. This occurs because of the absence of any $P d v$ work. In the more realistic simulations in Section 5.3 the gradients in the solution are non-negligible and this work term is no longer zero. The result is that for those situations there is a conversion of kinetic energy to internal energy which is indicated by an increased thermal energy.

Remarks on Sections 3.1

- This analysis ignores the mass of the optional pre-fill and the foam regions.

- Only the liner material is considered to be a conducting plasma.

- Only the mechanical work is included in the total energy equation. The contribution from Joule heating is considered negligible in this analysis.

- The axisymmetric assumption clearly does not hold for long time in physical Z-pinch implosions when either liner non-uniformities or nonuniform magnetic forces perturb the axisymmetric implosion. However, we will use source terms motivated by this limiting case analysis to produce prototype Z-pinch like implosions. It would appear that since the applied force, in this model, is always assumed to be in the negative radial direction this forcing term would not further enhance non-axisymmetric modes.

\subsubsection{Closed Form Solutions of Interest}

In the literature there are closed form solutions to Equation (16) for a number of special cases. The constant current case is presented here as well as the case where the radial position of the shell, $R(t)$, is assumed to be a power law relation and the corresponding current, $I(t)$, is derived which satisfies the thin-shell equation of motion.

Constant Current: For the case $I(t)=I_{\max }$, the following relationship for the radius as a function of time holds [1]

$$
\frac{t}{t_{A}}=\left(\frac{\pi}{2}\right)^{1 / 2} \operatorname{erf}\left[\left[\ln \left(\frac{R_{0}}{R}\right)\right]^{1 / 2}\right]
$$

This can be inverted to solve for the normalized implosion trajectory $R(t) / R_{0}$ as

$$
\frac{R(t)}{R_{0}}=\frac{1}{e^{\left[\operatorname{erf}^{-1}\left(\frac{t}{\tau_{i m p}}\right)\right]^{2}}}
$$

where $\tau_{i m p}=t_{A}(\pi / 2)^{1 / 2}$ is the non-dimensional implosion time. The velocity as a function of time is then given by

$$
V=-\frac{R_{0}}{t_{A}} \sqrt{2 \ln \left(\frac{R_{0}}{R(t)}\right)} .
$$

Power Law Radial Compression: For this case, a power law solution for the radius as a function of time is assumed to be given and then the current required to produce such a solution is derived. Assume a power law compression given by

$$
\frac{R(t)}{R_{0}}=\left[1-\left(\frac{t}{\tau_{\text {imp }}}\right)^{q}\right]
$$

for $q \geq 2$. The definition of the required current is easily derived from Equation (17) as

$$
I(t)=I_{\max } \sqrt{q(q-1)\left(1-\left(\frac{t}{\tau_{i m p}}\right)^{q}\right)\left(\frac{t}{\tau_{i m p}}\right)^{(q-2)}}
$$


with the velocity given by

$$
V=-\frac{q R_{0}}{\tau_{i m p}}\left(\frac{t}{\tau_{i m p}}\right)^{(q-1)} .
$$

As a special case of the power law radial compression (26), consider the case where $q=4$. In this case the current becomes

$$
I(t)=I_{\max } \sqrt{12}\left(\frac{t}{\tau_{\text {imp }}}\right) \sqrt{1-\left(\frac{t}{\tau_{\text {imp }}}\right)^{4}}
$$

which produces a radial implosion with normalized implosion trajectory of

$$
\frac{R(t)}{R_{0}}=\left[1-\left(\frac{t}{\tau_{i m p}}\right)^{4}\right]
$$

and velocity of

$$
V=-\frac{4 R_{0}}{\tau_{i m p}}\left(\frac{t}{\tau_{i m p}}\right)^{3} .
$$

In the results that follow in Section 5.2, current drive (27) is used in conjunction with a liner density profile that approximates a thin shell as a verification problem for our Euler equation model.

Linear Current: As a final example it is clear that for $t<<1$ the $q=4$ case produces a linear current drive behavior with

$$
I(t)=I_{\text {max }} \sqrt{12}\left(\frac{t}{\tau_{\text {imp }}}\right) .
$$

In some more realistic prototype implosions of Section 5.3, this linear current is used to drive implosions with

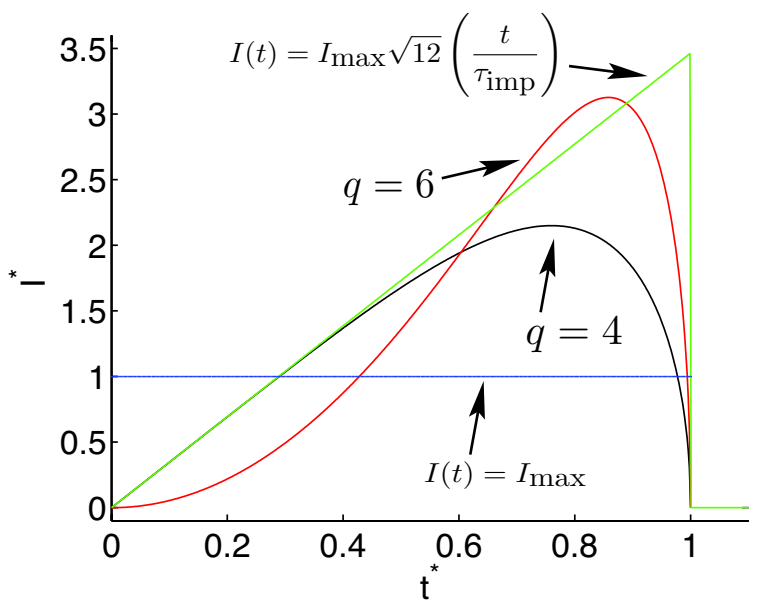

(a)

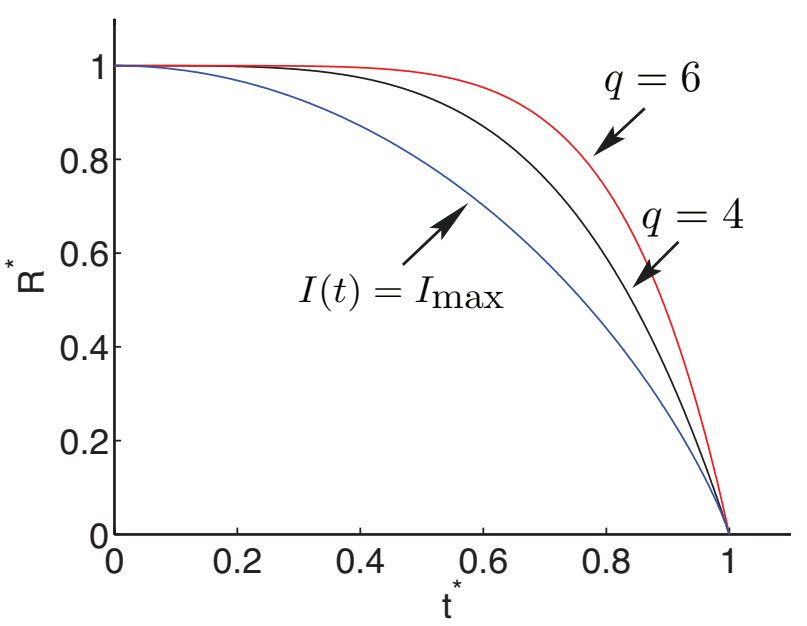

(b)

Figure 3: Various analytical results for thin-shell analysis. Frame (a) shows the current versus time while (b) shows the shell radius versus time.

pre-fill as well as problems which are intended to approximate magnetic Rayleigh-Taylor like effects. Using such a drive produces implosions that stagnate on the axis of symmetry in non-dimensional time $O(1)$ while maintaining the property that the current is rising as the material stagnates. Finally, Figure 3 presents a few forms of current drives and normalized implosion trajectory. that are solutions of the thin-shell analysis. 
Various forms of the power law current model can be used to produce qualitative correspondence to actual Z-pinch experiments (see e.g. Figure 6 in [12]).

Remarks on Section 3.1.1

- The idealized solutions are only for the thin-shell model (16) and they have the same limitation as described above in Remarks on Section 3.1

- These solutions predict an infinite compression ratio and an infinite magnitude Lorentz force at the origin which is unphysical. In practice the equations are only used at points which lie outside some $\epsilon$ radius of the origin where $\epsilon$ is some small positive number.

- Other solutions of the thin shell model are also possible and produce a nonlinear equation for the normalized implosion tragetory as a function of time.

\section{Description of Models}

This section briefly describes two types of models for a magnetic drive source term for the momentum and energy equations based on the thin-shell models developed above in Section 3. These Lorentz body force and work terms are used to produce a Z-pinch like prototype implosion problem that can be used in the development and evaluation of shock-hydrodynamics simulation tools. Initially a modified form of the thinshell Lorentz force term which removes the singularity at $r=0$ is presented. The model then adds a scalar transport equation to the conservation law system which is used to define a region approximating a "current sheet" that localizes the action of the Lorentz force term.

\subsection{A Model for the Magnetic Drive $\mathrm{J} \times \mathrm{B}$ Force}

One significant issue in the simple thin-shell analysis is the singularity of the solution at $t=\tau_{\text {imp }}$. This singularity produces an infinite magnitude for the Lorentz force as well as the strength of the magnetic field at $r=0$, which produces an infinite velocity and kinetic energy. In order to avoid this behavior, the thin-shell Lorentz force term is modified to produce an effective limiting compression ratio as

$$
\mathbf{f}_{\lambda l}^{*}=\lambda \rho^{*}\left(\frac{I(t)}{I_{\max }}\right)^{2} \frac{1}{r_{e f f}^{*}} \hat{\mathbf{e}}_{r} .
$$

In this term, the non-dimensional $r$-coordinate $r_{\text {eff }}^{*}$ is used to define the strength of the source term locally and to remove the singularity at $r=0$ with the definition

$$
r_{e f f}^{*}=\max \left(r / R_{0}, r_{\min }^{*}\right) .
$$

Further, the scalar $0 \leq \lambda\left(r^{*}, t^{*}\right) \leq 1$ is used to control the magnitude and localize the application of the non-dimensional Lorentz force term. In the simulations presented in this work $r_{\min }^{*}=1.0^{-4}$ is typically used. Notionally the scalar $\lambda\left(r^{*}, 0\right)$ is considered to define the initial "current sheet" and to localize the application of the source term during the evolution of the implosion. Therefore by including this force term, in a suitably non-dimensionalized form of the Euler equations, a parameterized set of prototype magnetically driven shock-hydro problems can be developed. In this way a typical Euler code and a proposed shock-hydro implementation which is based on this Euler code can be evaluated on prototypical Z-pinch like implosions without the addition of any electromagnetics capabilities.

Finally, it should be noted that while the source term (31) has a discontinuous first derivative, this discontinuity is only a source term in the equations and therefore does not require higher order continuity. Additionally, this relationship preserves the behavior of the Lorentz term from the thin-shell analysis with an effective compression ratio of $\alpha_{\text {min }}=r_{\text {min }}^{*}$ for $t \geq \tau_{i m p}$. Figure 4 presents a schematic diagram showing a cross-section of a geometry where a sheath-like "current sheet" is shown at time $t=0$. 




Figure 4: Schematic cross-section of the initial geometry where the sheath region is shown.

\subsection{A Combined Scalar Transport Model for $\mathrm{J} \times \mathrm{B}$}

To implement this model in a single temperature Euler system, the definition of the source term, $\mathbf{f}$, in (2) is assumed to be given by the non-dimensional Lorentz force (31) with the interpretation of $\rho$ as a density per unit length which has been non-dimensionalized by the initial linear density $\rho_{L}^{0}$. The Euler single temperature system with the advected scalar, $\lambda$, is then defined by

$$
\begin{gathered}
\mathbf{U}=\left[\begin{array}{c}
\rho \\
\rho \mathbf{v} \\
E \\
\rho \lambda
\end{array}\right], \quad \mathbf{F}=\left[\begin{array}{c}
\rho \mathbf{v} \\
\rho \mathbf{v} \otimes \mathbf{v}-\mathbf{T} \\
E \mathbf{v}-\mathbf{T} \cdot \mathbf{v}+\mathbf{q} \\
\mathbf{v} \rho \lambda
\end{array}\right] \\
\mathbf{S}=\left[\begin{array}{c}
0 \\
-\mathbf{f} \\
-\mathbf{f} \cdot \mathbf{v}+Q_{\text {rad }} \\
0
\end{array}\right]
\end{gathered}
$$

A clear advantage of tracking an advected scalar in this way is that the Lorentz source term $\mathbf{f}=\mathbf{J} \times \mathbf{B}$ can be evaluated by direct evaluation of $r_{\text {eff }}^{*}$ in the denominator of Equation (15) at every point in the fluid. That is to say that one can consider $\lambda$ to define a current sheet at $t=0$ and an active region for the Lorentz force term in the evolution of the implosion of the liner material. Thus by specifying $I(t)$ the forcing term magnetically drives the Z-pinch like implosion with the required $1 / r$ behavior in the active region where the Lorentz force term per unit length is non-zero.

\section{$5 \quad$ Numerical Results}

To give some idea of the applicability of the models developed in this paper, a number of numerical results using the scalar transport localization of Section 4.2 are presented. Results are obtained through the use of a flux-corrected-transport (FCT) flow solver and explicit time stepping which has been built into a composite overlapping grid framework.

\subsection{Numerical Method}

The numerical method used for the solution of the conservation law system (1) which has been utilized in this work is built within an overlapping grid solver framework. An overlapping grid is a collection of grids, 


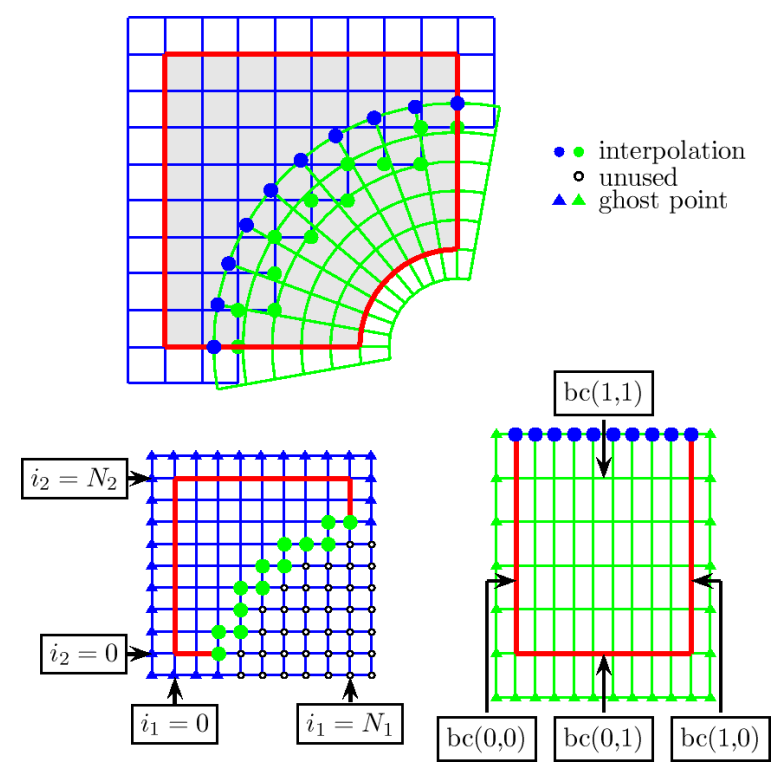

Figure 5: (Color) The top view shows an overlapping grid consisting of two structured curvilinear component grids. The bottom views show the component grids in the unit square parameter space. Grid points are classified as discretization points, interpolation points (green or blue dots), or unused points (open disks). Ghost points (green or blue triangles) are used in the application of boundary conditions.

in this case all logically rectangular, that cover the computational domain in some overlapping fashion and communicate at this overlap through interpolation. A simple example of an overlapping grid is demonstrated in Figure 5 which shows an overlapping grid consisting of a blue Cartesian grid and a green annular grid. In this figure one can see the blue and green dots which represent the interpolation points where the solution is communicated from one grid to the other, as well as the open circles which represent unused points where no computation needs to be performed. Also it should be noted that for our application all grids are logically rectangular which leads to increased computational efficiency. Extensive use is made of adaptive mesh refinement (AMR) so that highly resolved grids are used only near regions of rapid change such as shocks or contact surfaces. This combination of logically rectangular grids with AMR makes for an extremely efficient method which enables very finely resolved computations. A more detailed discussion of overlapping grids and AMR can be found in $[15,16]$.

Within this overlapping grid context, a flux-corrected transport (FCT) algorithm is used for the flow solver $[17,18]$. The basic FCT algorithm was originally developed in $[19,20,21]$ and has seen much success since that time. The FCT algorithm used here follows closely the ideas in $[22,23]$ but has been extensively modified for operation in the overlapping grid context as well as to produce more robust behavior in the presence of very strong shocks [18]. The end result is a monotone scheme which necessarily exhibits low order convergence near extrema or discontinuities in the characteristic variables, but which converges at second order for large regions of the flow which are smooth. This type of non-linear stability result is critical when simulating flows with extreme jumps such as the 7 order of magnitude jumps in density experienced in our simulations.

\section{$5.2 \quad$ Asymptotic Thin-shell Implosion Problem}

This section presents two challenging magnetically driven implosion problems that are intended for quantitative and qualitative evaluation of shock-hydro algorithms intended for z-pinch type simulations. The problems are based on the classical 0-D thin shell cylindrical implosion analysis (Section 3.1). This analysis produces an analytic asymptotic solution to the ideal MHD equations. In the development of a computational capability for modeling Z-pinch systems these problems can be used in the following ways. First a 


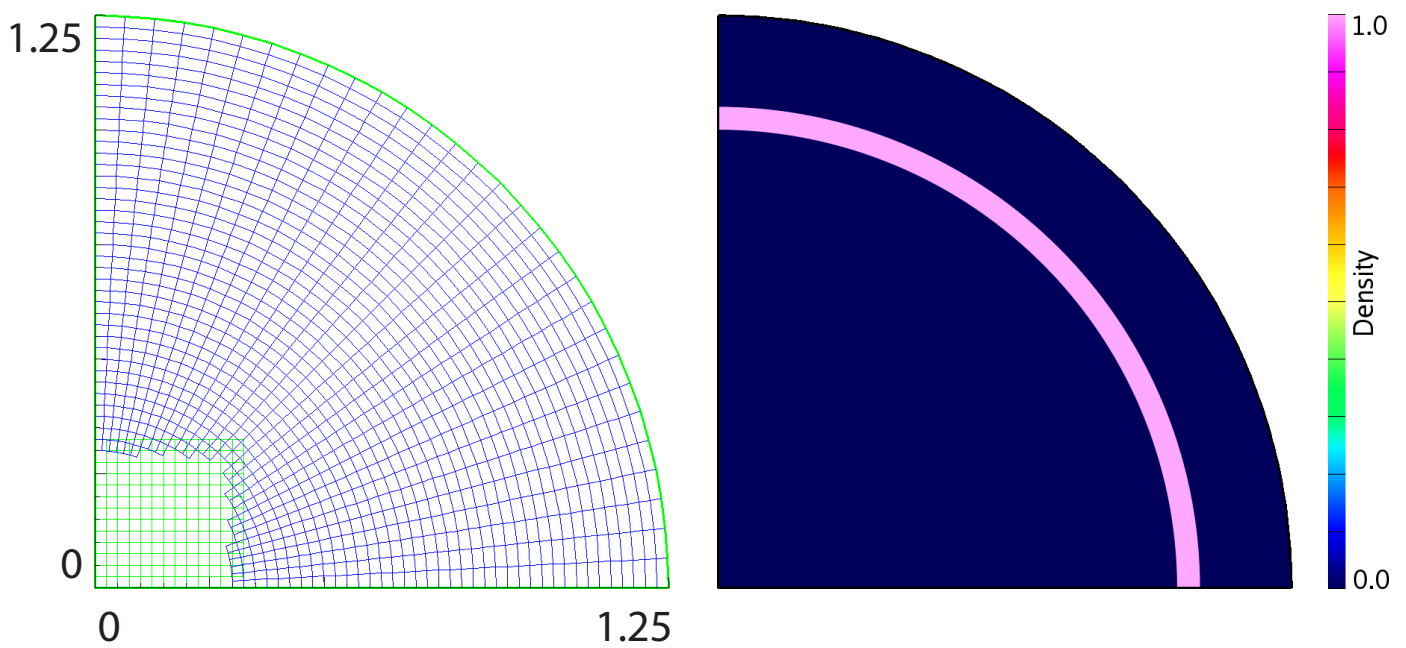

Figure 6: (Color) Left: Computational grid used for the magnetic implosion simulations. Here an annular grid (blue) is used for much of the domain with the singularity covered by a square patch (green) in the center. Right: Initial condition of density for for the highest resolution simulation of the simple implosion. Notice the thin shell of high density surrounded by nearly rarefied regions interior and exterior. The non-dimesnional values for these densities are $\rho_{L}=1.0, \rho_{\text {pre-fill }}=1.0 \times 10^{-6}$ and $\rho_{\text {exterior }}=5.0 \times 10^{-7}$.

quantitative assessment or verification of the solution of the ideal Euler equations with source terms can be made in both $(r, z)$ and $(x, y)$ geometries as demonstrated in Sections 5.2.1 and 5.2.2. This simple problem would constitute a necessary condition for Euler solvers intended to model z-pinch type systems. Additionally these problems can be used to qualitatively evaluate shock-hydro algorithms for robustness in handling very large density jumps, the development of excess numerical diffusion at contact surfaces, the maintenance of symmetry for cylindrical implosions, and the ability to handle accurately and robustly the singular point at the origin in the $(\mathrm{x}, \mathrm{y})$ converging geometry. In this context this type of problem can be used as a decision point to make a go or no-go assessment of the proposed shock-hydro algorithm.

\subsubsection{Thin-shell Implosion in the $x-y$ Plane}

The simple $(x, y)$ implosion problem uses the initial geometry presented in Figure 1 . In order to give a reasonable sense of the expectations of such a model, the non-dimensional initial conditions are choosen with an interior density of $\rho_{\text {pre-fill }}=1.0 \times 10^{-6}$, a liner density of $\rho_{L}=1.0$, and an exterior density of $\rho_{\text {exterior }}=5.0 \times 10^{-7}$. The initial pressure throughout the domain is $p_{0}=1.0 \times 10^{-6}$ and the flow is initially stagnant. The current drive is chosen to be $I(t)=\sqrt{12\left(1-t^{4}\right) t^{2}}$ which results in the power law implosion $R(t)=1-t^{4}$. In order to give a reasonable intuitive sense for these initial conditions as well as the computational domain where the simulation is carried out refer to Figure 6 . The base computational grid which is used for these simulations is shown in Figure 6. Here an annular grid (blue in the figure) is used over much of the domain with the singularity at the origin covered by a square grid (green in the figure). The grid spacing in the radial direction as well as for the center square patch is chosen to be $h=0.025$, and the average grid spacing in the azimuthal direction for the annular grid is $h \approx 0.059$. Solid slip-wall boundary conditions are applied at the left and bottom, and an inflow condition is applied at the outer curved boundary. A series of results for a shell of fixed thickness $\Delta=0.05$ is presented using increasing amounts of AMR. For the initial resolution no AMR is used. The second resolution uses one additional level of AMR with a refinement factor of 4 in each direction giving the effective resolution of $h=0.00625$. The final resolution takes 2 additional levels of AMR each with a refinement factor of $4(h=1 / 640)$. The initial condition for density for the finest resolution simulation is presented in Figure 6 . Here the thin ring of conducting material is seen as pink which represents $\rho=1.0$ and $\lambda=1.0$. Interior and exterior to this ring the density is so low $\left(\approx 10^{-6}\right)$ that one cannot distinguish it from a pure vacuum using the included 



Figure 7: (Color) Density for the highest resolution simulation at $t=0.5$ (right), and $t=0.9$ (left). The thin shell is seen to implode in a remarkably symmetric fashion even after the transition from the annular grid to the square center patch.

colorbar. Figure 7 shows two snapshots of the density at $t=0.5$, and $t=0.9$. These two images show the imploding conducting shell which appears to remain quite cylindrically symmetric even after its transit from the annular grid to the center square patch. It can be seen, particularly at the later time $t=0.9$, that the thin shell model is clearly approximate. The most obvious indication of this is the fact that the peak density now has a much higher value $\rho_{\max } \approx 1.75$, which results from non-uniformities in the simulation as well as because the shell here is not infinitely thin and compressional effects are induced that are not accounted for in the thin-shell model of Section 3.

In order to evaluate the degree to which the scalar transport model of Section 4.2 agrees with the thin shell model, Figure 8 presents the shell radius, $R(t)$, and the shell velocity $V(t)$. The values for these plots are obtained from the simulation results but because we are dealing with simulation results obtained using a capturing code, the selection of the location, and hence velocity, for the imploding shell is open to interpretation. In these results the shell location at a given time is defined by the location of the computational cell nearest to the origin whose density is $1 / 2$ of the maximum density at that time. From this cell the radius and velocity are selected and used in the plots. In these plots the clustering of the data about the analytic solution demonstrates good agreement of the simulation with the predicted behavior for the thin shell model. One can also see the solution for finer mesh resolutions converging to the analytic solution at all times.

Figure 9 presents an attempt to quantify, with error bars, a measure of the error in the finest mesh computation using the definition of $R(t)$ and $V(t)$ as described above. The plots here show three locations; one as before, one with density at $10 \%$ of the maximum value, and one with density $90 \%$ of the maximum value. From these locations the radius and velocity are calculated and used as a bracket to indicated possible measurement error. These plots show the excellent agreement of the simulation with the analytical solution.

\subsubsection{Thin-shell Implosion in the r-z Plane}

As a further investigation of the model, behavior is investigated for an axi-symmetric geometry. Here the simulation is carried out in the r-z plane with an axi-symmetric assumption which is a common mode of operation within the Z-pinch implosion community. As before, an initial investigation is performed in order to gauge agreement to the thin shell approximation for the exact solution $R(t)=1-t^{4}$ and $V(t)=4 t^{3}$. For this investigation the initial grid shown in Figure 10 is used and has resolution $\Delta r=\Delta z=0.025$. It should be noted that a mesh of only 4 cell vertices in the $z$-direction is used because there is absolutely no variation in the $z$-direction for the duration of the simulation and so the width of the grid has no effect on simulation results. By choosing a thin grid the computational cost is reduced making more finely resolved 

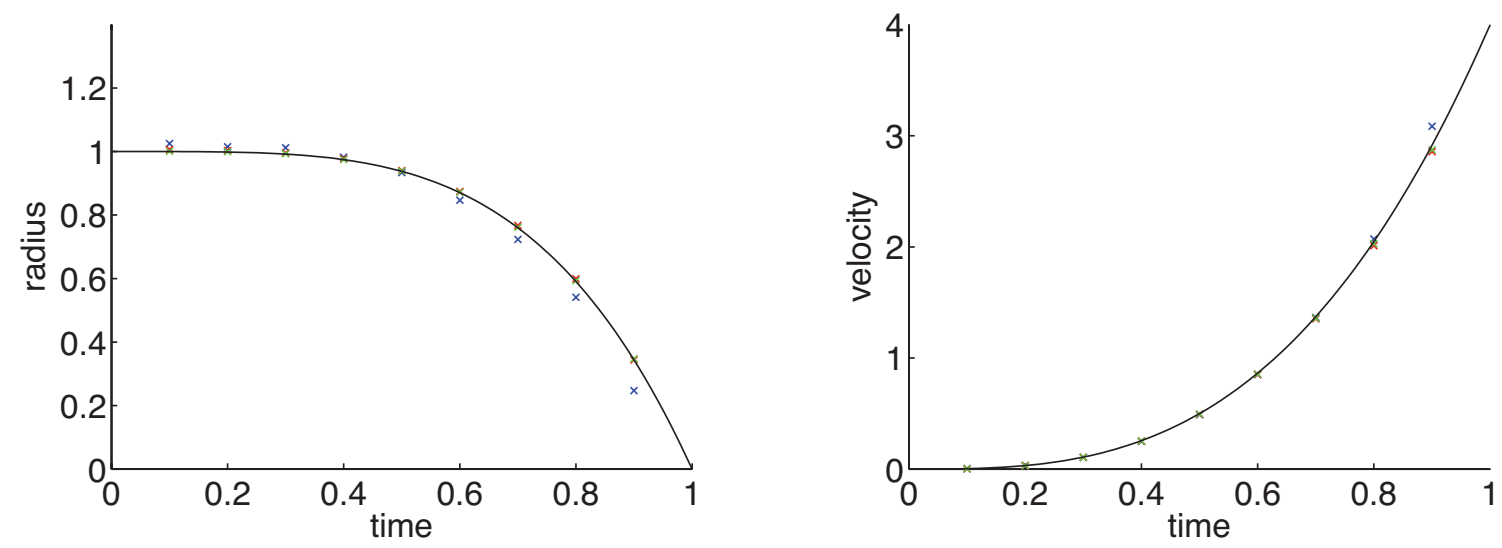

Figure 8: Shell radius against time (left) and shell velocity against time (right). In these plots the black curve represents the exact solution $R(t)=1-t^{4}$ and $V(t)=4 t^{3}$, the blue x's represent the solution with no AMR, the red x's represent the solution with one level of AMR, and the green x's represent the solution with two AMR levels. We can see the convergence of the solution to the exact thin shell prediction upon increasing resolution.
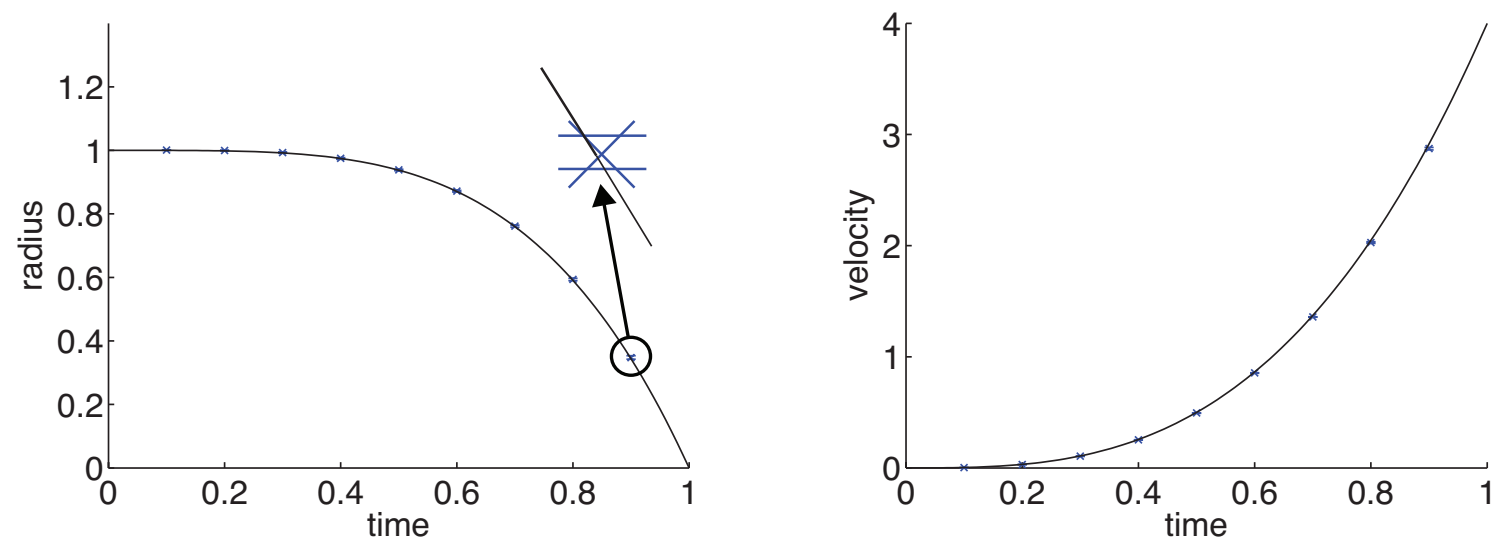

Figure 9: Shell radius against time (left) and shell velocity against time (right). In these plots the black curve represents the exact solution $R(t)=1-t^{4}$ and $V(t)=4 t^{3}$. The blue x's represent the solution with 2 levels of AMR and the error bars indicate the uncertainty in measurement for the given quantity as outlined in the text. Very good agreement between the numerics and analytic solution is seen and the error bars nicely bracket the analytic solution. 


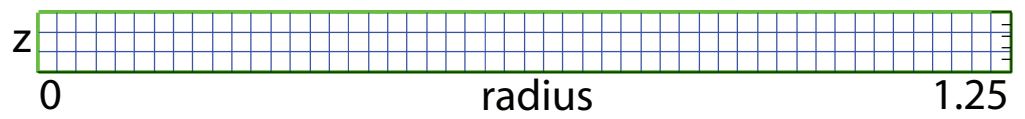

Figure 10: Base computational grid for simulation in the r-z plane used to compare with the thin shell model. The axis of symmetry is where the radius $r=0$. This very thin mesh is used because no z-variation is experienced for the duration of the simulation and so it is of no advantage to use a larger mesh.



Figure 11: (Color) Color contours of density at $t=0,0.5$, and 0.9 (top to bottom) for simulation in the $\mathrm{r}-\mathrm{z}$ plane. Here it is clear that there is no variation in the $\mathrm{z}$-direction as the simulation progresses.

simulations more practical. As before, the densities are set such that a thin shell is approximated. Thus $\rho_{\text {pre-fill }}=1.0 \times 10^{-6}, \rho_{L}=1.0$, and $\rho_{\text {exterior }}=5.0 \times 10^{-7}$. Figure 11 shows the initial condition and two later times for the density. Here the base computational grid in Figure 10 is used with two additional levels of factor 4 AMR refinement and the imploding shell is clearly seen as it approaches the axis of symmetry. Again it is informative to plot the radius and velocity of the imploding shell as a function of time to compare with the thin shell analytic model. As before the radius and velocity are determined from the cell closest to the axis of symmetry where the density has risen to $50 \%$ of the maximum value at that time. As well a measurement error estimate is included by determining the cells where the density has attained $10 \%$ and $90 \%$ of the maximum value. Figure 12 shows these results as a function of time. As was the case for the simulations in the $\mathrm{x}-\mathrm{y}$ plane, these simulations in the r-z plane show good agreement with the analytic solution. The error bars in both radius and velocity nicely bracket the solution at all times demonstrating the high level of agreement between the simulation and the thin shell model.

\subsection{Initial Extensions to More "Realistic" Simulations}

In this section, initial results are presented that correspond to a number of Z-pinch like prototype problems. The prototype problems are intended to illustrate different aspects of applying the magnetic drive source term to produce liner implosions with plasma pre-fill and dynamic hohlraum effects [13, 14]. The intent of these problems is to more aggressively test proposed shock-hydro algorithms since effects such as vorticity due to Rayleigh-Taylor and/or Richtmyer-Meshkov type instabilities will be generated. The result is that these tests will require the use of remapping in proposed arbitrary Lagrangian Eulerian (ALE) methods and therefore test the Eulerian capabilities of such codes.

The presented problems represent Z-pinch type implosions with the choice of parameters guided by values found in the literature for actual pinches and pinch simulations. For instance Section 5.2 presents the thin shell model with a density jump of 6 orders of magnitude and an initial pressure of $1 \times 10^{-6}$ with a resultant sound speed of $c=\sqrt{\gamma}$ in the pre-fill region which is on the same order as the implosion time. Indeed this is critical in order to obtain accurate agreement with the thin shell approximation, but as is stated in [24], a real wire array implosion is preceded by significant wire ablation which introduces substantial amounts of material into the pre-fill region before implosion even begins. In that work it is shown that near the time implosion actually starts $\rho_{\text {pre-fill }} \approx 0.05$. Figure 14 shows simulation results using this value for the pre-fill, $\rho_{L}=1.0, \rho_{\text {exterior }}=0.025$ and $p_{0}=0.01$. The base computational grid has a mesh spacing of $h \approx 0.0025$ 

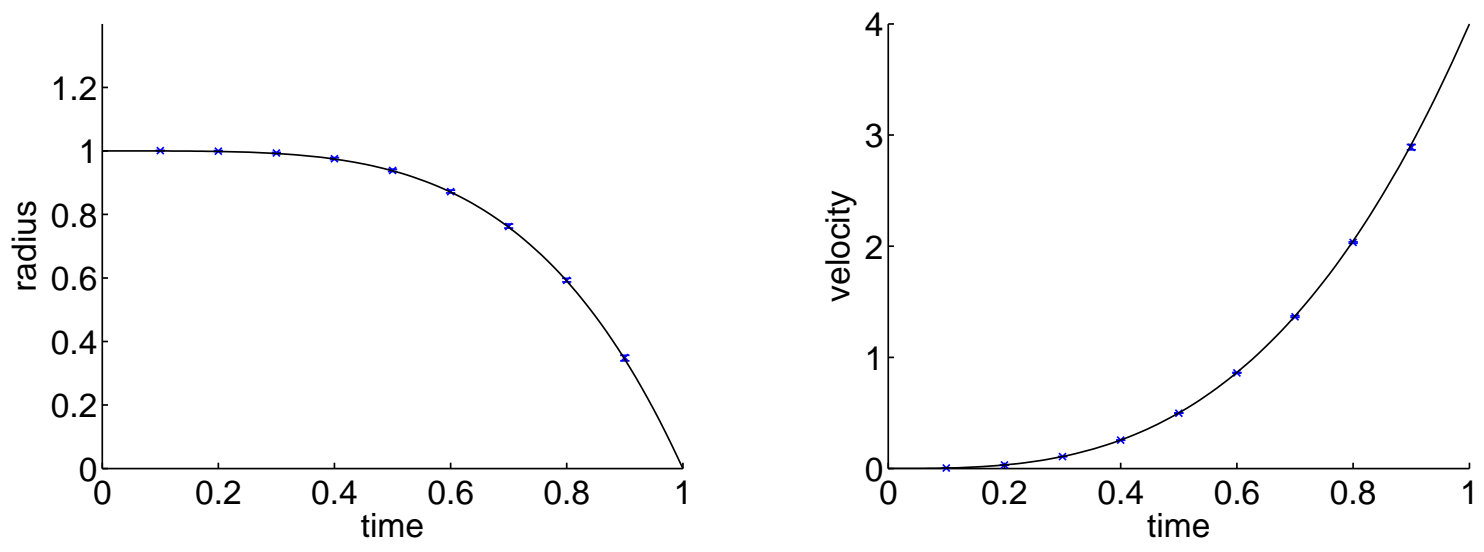

Figure 12: Shell radius against time (left) and shell velocity against time (right). In these plots the black curve represents the exact solution $R(t)=1-t^{4}$ and $V(t)=4 t^{3}$. The blue x's represent the solution with 2 levels of AMR and the error bars indicate our uncertainty in measuring the given quantity. As before the simulation results are seen to be in very good agreement with the analytic solution.

and uses two additional levels of factor 4 AMR to give an effective grid resolution of $h \approx 0.0015625$. As previously stated, these simulations use the linear current drive (30) that remains active during stagnation of the material on axis. Clearly $t_{\text {implosion }} \neq 1$ for this case because substantial material now exists in the pre-fill region and the linear current drive $I=\sqrt{12} t$ is used rather than $I=\sqrt{12\left(1-t^{4}\right) t^{2}}$. The final image of Figure 14 has the compressed liner partially past the cylindrical-to-square mesh transition region with reasonable symmetry maintained by the numerical method.

As another variation, perturbations to the initial geometric configuration of the shell are introduced for a prototype implosion in the $r-z$ plane. Figure 15 shows a numerical Schlieren of the time evolution of the initial conditions where a sinusoidal perturbation to the initial interface has been introduced. This perturbation is intended to promote the growth of a particular unstable Raleigh-Taylor mode and thus create significant structure as the liner nears stagnation. Here the perturbation has amplitude 0.005 and period 0.125 and has been introduced along the inner boundary of the conducting shell. The initial pressure distribution, $p_{0}=0.01$, is selected to promote growth of R-T spikes ahead of the liner implosion at a sufficient rate so as to view their effect before stagnation. The instability evolves from the initial conditions on the right to the stagnation on axis on the left. The FCT AMR solution provides a very well resolved simulation of multiple unstable modes resulting in a complex pattern of R-T growth with complex interaction of shock waves at stagnation. It should be noted that the evolution of the R-T instability is qualitatively different from an actual Z-pinch system in which spikes lag behind the remaining liner material due the larger current flow in this contiguous material sheet (for example see $[25,11]$ ). This is due to our simplified model assumption that defines the "current sheet" by the scalar $\lambda$ that cannot adequately model the preferential physical current flow through the contiguous liner material over the penetrating spikes. However the magnetic force term does produce spikes and sheets of material developed by the R-T effects and R-M instabilities as the strong-shock interacts with the trailing liner material sheet. These later stages have some qualitative similarities to actual Z-pinch implosions which gives indication to why such a simple testing procedure can be very beneficial in benchmarking the flow portion of simulation tools. Alternatively in Figure 16 a sinusoidal perturbation with amplitude of $2.5 \%$ in the density is introduced in the conducting shell and an initial pressure, $p=0.01$, is selected to promote growth of R-T spikes ahead of the liner implosion. Clearly the time evolution here is very similar to that shown in Figure 15 with the main difference being that the onset of significant spikes is delayed for the density perturbation as compared to the geometric perturbation. Of course this is expected because for both cases it is the mechanical work of the pre-fill material which provides the forcing of the instability. In the case of the density perturbation time is required to generate the initial geometric perturbation and thus the visible geometric growth of instabilities is delayed. In any case the late time dynamics at stagnation are similar for both cases. 

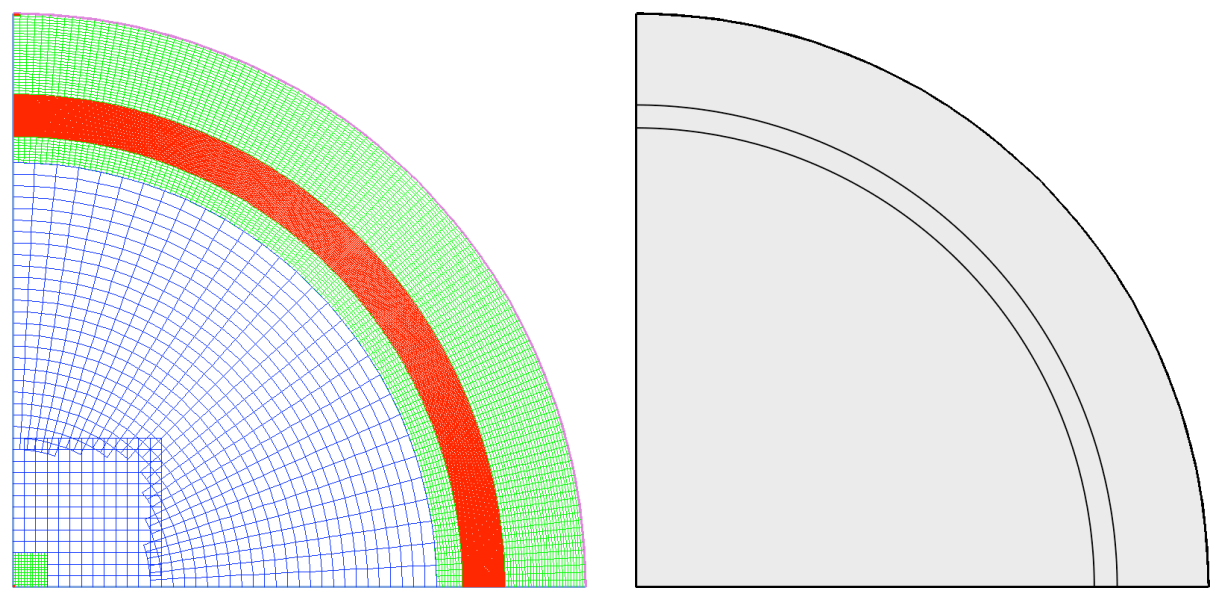

Figure 13: (Color) Numerical Schlieren images of the perturbed initial condition for the dynamic implosion (right) and the initial AMR grid structure to capture the density jump (left). Here the blue represents the base grid, green the second level, and red the finest.


Figure 14: Implosion with $\rho_{\text {pre-fill }}=0.05$ and $p_{0}=0.01$ at $t=0.7$ (right) and $t=1.0$ (left). Plotted here is a numerical Schlieren which indicates regions of high density gradient. 

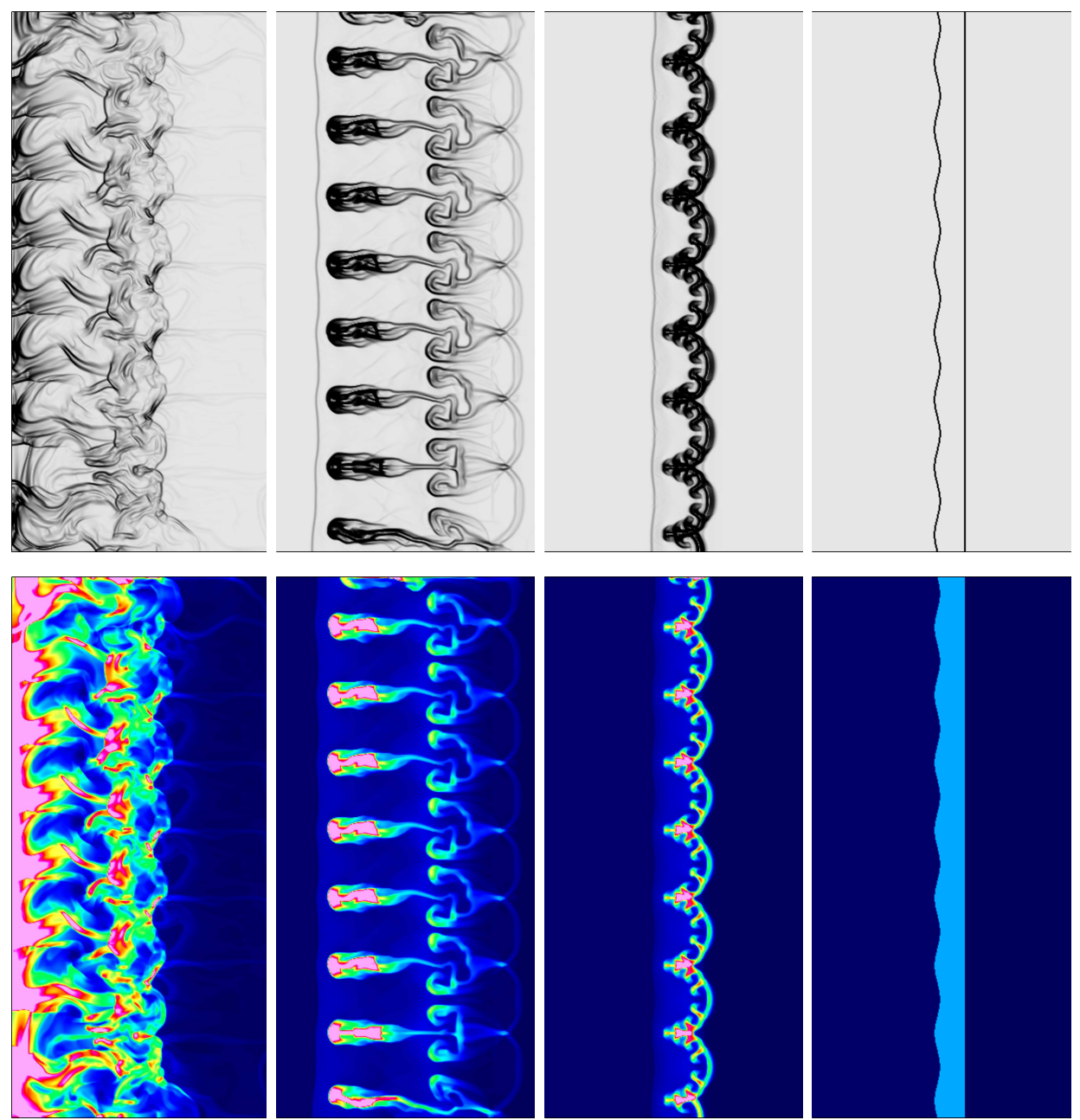

Figure 15: (Color) A prototype Rayleigh-Taylor instability for a perturbed liner geometry for the magnetic implosion in $(r, z)$. An initial pressure distribution to promote growth of $\mathrm{R}$ - $\mathrm{T}$ spikes ahead of the liner implosion has been selected. The instability evolves from the initial conditions on the right to the stagnation on the axis on the left. The upper images are a numerical Schlieren and the lower images show the density at times (from left to right) $t=1.2, t=1.0, t=0.8$, and $t=0.0$. 

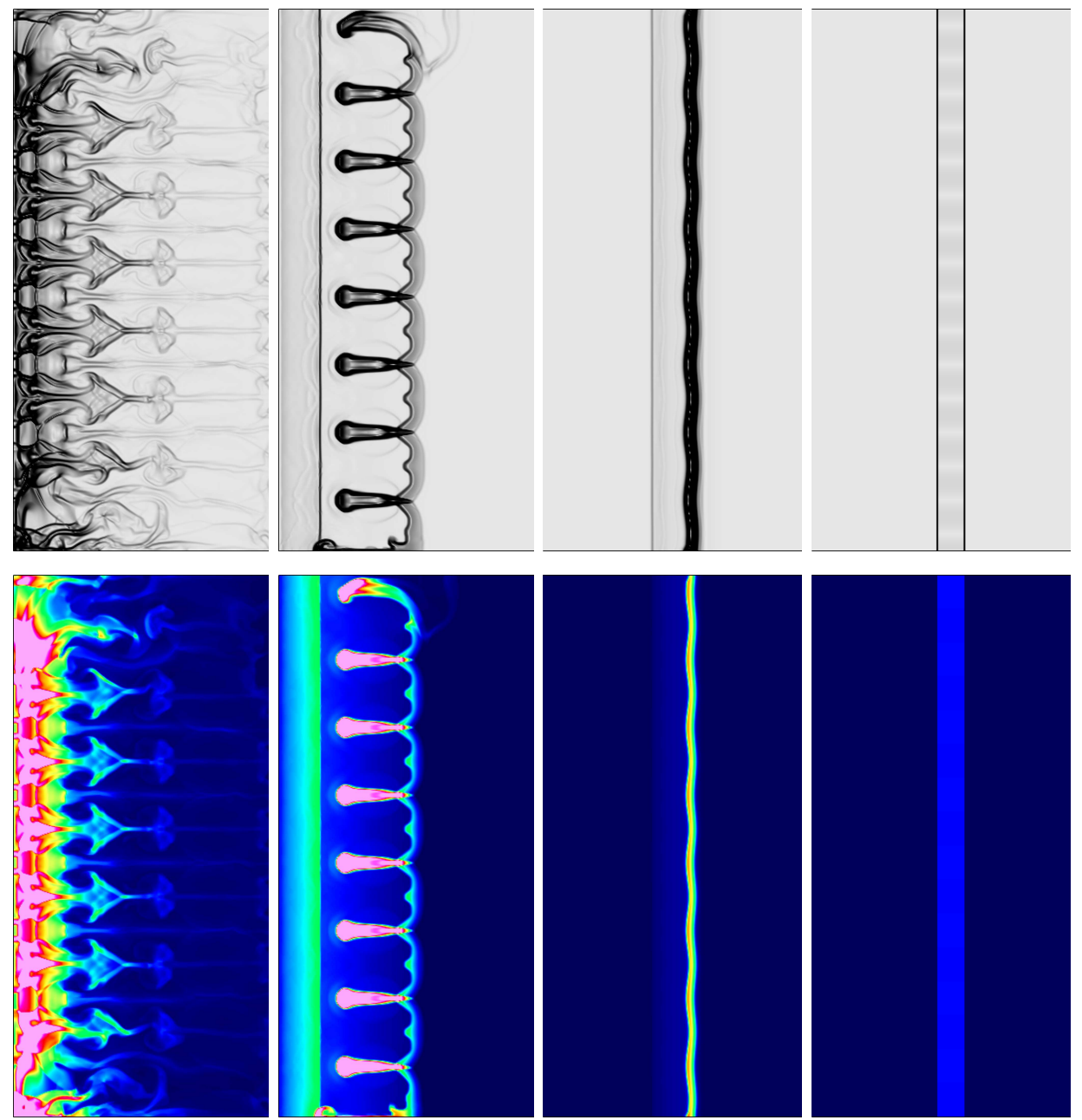

Figure 16: (Color) A prototype Rayleigh-Taylor instability for a perturbed density configuration for the magnetic implosion in $(r, z)$. Here a sinusoidal perturbation of amplitude $2.5 \%$ has been introduced in the density of the conducting shell, and an initial pressure $p=0.01$ is selected to promote growth of R-T spikes ahead of the liner implosion. The instability evolves from the initial conditions on the right to the stagnation on the axis on the left. The upper images are for a numerical Schlieren and the lower images represent density at times (from left to right) $t=1.3, t=1.1, t=0.8$, and $t=0.0$. 

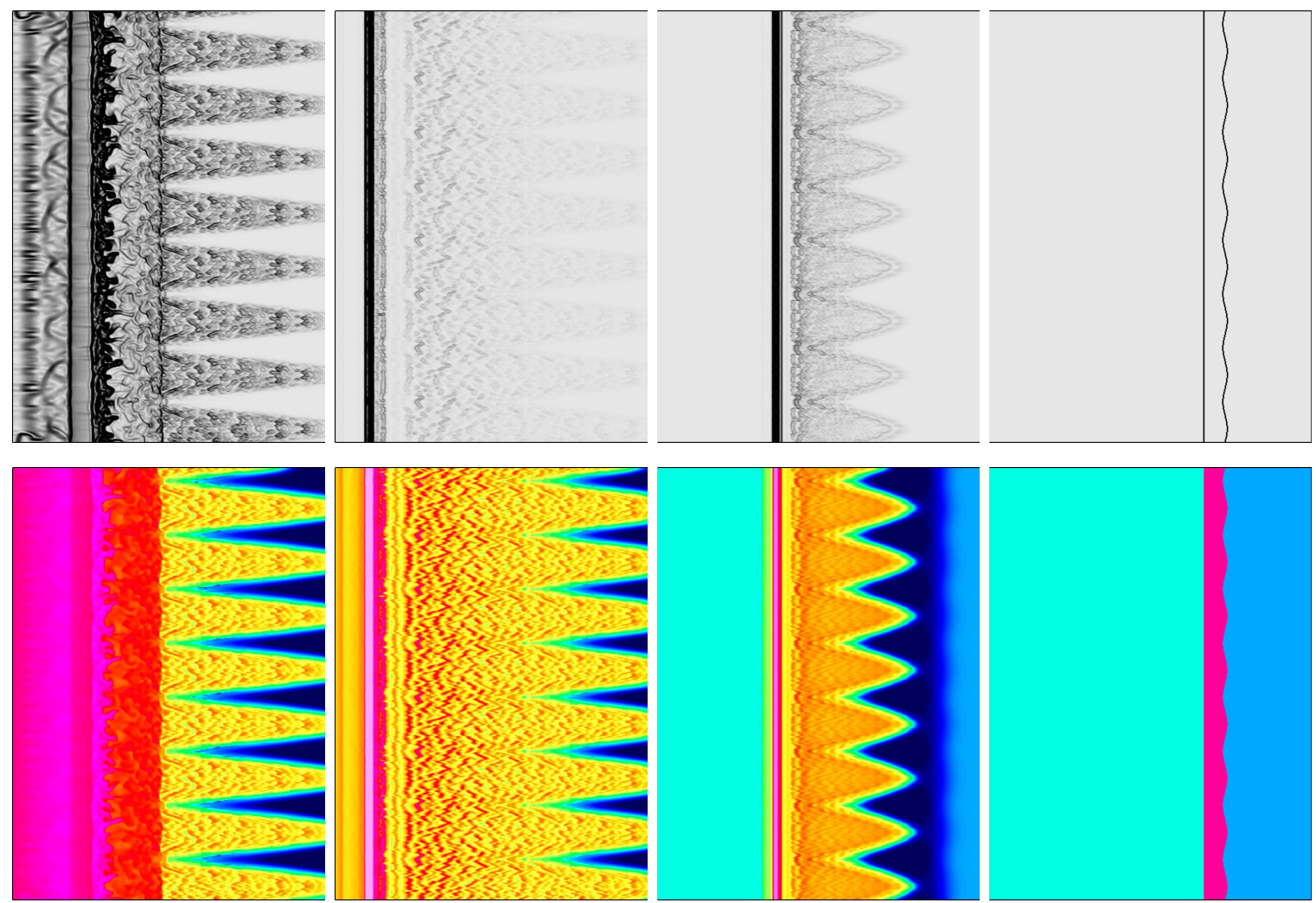

Figure 17: (Color) A prototype Rayleigh-Taylor instability for a perturbed density configuration for the magnetic implosion in $(r, z)$ which has been designed to promote the formation of trailing mass. Here a sinusoidal perturbation of amplitude $2.5 \%$ has been introduced on the trailing side of the liner and the initial pressure distribution is chosen to inhibit growth of R-T spikes ahead of the liner implosion. The current sheet is initialized Gaussian function centered at mid-plane of the liner. The upper images show a numerical Schlieren and the lower images the logarithm of the density at times (from left to right) $t=1.02$, $t=0.98, t=0.8$, and $t=0.0$.

An important issue in actual Z-pinch implosions is the dynamics of trailing mass [26]. Figure 17 presents a prototype density configuration for the magnetic implosion in $(r, z)$ that has been designed to promote the formation significant trailing mass. Here a sinusoidal perturbation of the geometry of the liner at the trailing edge has been introduced with an amplitude of $2.5 \%$ and the initial pressure distribution has been selected to inhibit growth of R-T spikes ahead of the liner implosion $\left(p=1.0 \times 10^{-6}\right)$. In addition, the current sheet as been initiated as a Gaussian function centered at the mid-plane of the liner with the specific form $\lambda=\exp \left[-\left(\frac{1.025-r}{.025}\right)^{2}\right]$. This particular choice results in approximately a $50 \%$ drop in the Lorentz force at the edges of the liner. Clearly the effect of the initial localized current sheet with diminished Lorentz force near the trailing edge of the imploding liner is to promote the development of trailing mass in the implosion.

In this section a series of representative implosion geometries and initial density distributions has been presented with the intent to produce implosion dynamics of interest in Z-pinch type systems. As presented these problems can all be modeled with a minimally modified Euler system solver. In the next section a brief example of what can be expected when including additional physics in the model is presented by considering a simple radiation emission model. 


\section{An Example of Additional Physics in Euler: A Simple Radiation Model}

As described above a significant motivation for the phenomenological magnetic drive source term model is to allow the evaluation of shock-hydrodynamics algorithms intended to be used as a base to build Z-pinch type simulation capabilities. This brief section presents the results of an initial study including additional physics in the Euler shock-hydrodynamics simulations, here a simple radiation emission model. As discussed in the introduction one important advantage of developing this simple Lorentz force term to drive "Z-pinch like" implosions is that hydrodynamic and radiation-hydrodynamic algorithms can be tested, evaluated and stressed on nearly realistic challenging implosions. Below is an example of such a problem using a very simple radiation emission model. Even with the use of such a simplified model, the results produced by the Euler system solver with the $\mathbf{J} \times \mathbf{B}$ source term model produce power pulses with qualitative similarities to experimental and full computational MHD results found in the literature (see e.g. Figure 9 in [12]).

One of the simplest radiation emission models one might consider is

$$
Q_{\text {rad }}=\sigma T^{4}
$$

where $T$ is the temperature obtained from the equation of state (8) and the caloric equation of state (9). In the literature there are many different forms for $\sigma$ depending on the model's intended regime, the specific material under consideration, etc. For simplicity this work chooses the rather simple form

$$
\sigma=\bar{\sigma} \rho \frac{1}{T^{2}}
$$

where $\bar{\sigma}$ is a constant chosen here to be $\bar{\sigma}=100.0$. The radiation emission is set to be active only in the liner material by multiplication with $\lambda$ so the final radiation source term becomes

$$
Q_{\text {rad }}=\bar{\sigma} \lambda \rho T^{2} .
$$

Obtaining resolved radiation profiles in an actual Z-pinch simulation is a tremendously challenging task due both to the complexity of the physically realistic radiation models as well as the mesh resolution requirements. With this difficulty in mind, this section presents two convergence studies where different aspects of this difficult regime are highlighted. This will illustrate some of the difficulties one might expect to encounter as well as build an intuitive understanding of the resolution requirements for generating radiation profiles.

The first problem is a pinch in the $r-z$ plane with some small amount of pre-fill material and a stagnation point off axis at $r=0.1$ as for example on a rod. Here the pre-fill and exterior densities are $\rho_{\text {pre-fill }}=$ $1 \times 10^{-3}, \rho_{\text {exterior }}=5 \times 10^{-4}$ and the initial pressure is $p=1 \times 10^{-5}$. A linear current drive $I=\sqrt{12} t$ is used so the current is rising as the material stagnates and radiates. The study is performed at a variety of uniform mesh resolutions corresponding to $h=1 / 160,1 / 320,1 / 640,1 / 1280,1 / 2560$ and $1 / 5120$ where $h$ is the grid spacing in the $r$ and $z$ directions. Figure 18 presents the radiation output and the total radiated power as a function of time for these resolutions and it is clear that the pulse is well behaved and largely converged at the finest resolution $h=1 / 5120$.

In order to gauge convergence, consider the convergence rate in two separate norms. The first and perhaps simplest is to look at is the extrapolated convergence rate as considered by Roy in [27]. In this analysis the final three data points for total radiated power from Table 1 are considered and an order of convergence is obtained, as in [27], from this data. For this data the convergence rate is judged to be $\kappa \approx 1.26$ for the total radiated power at $t=1.0$. For the second measure consider the self convergence in $L_{1}$ of individual radiation pulses to the finest computation $h=1 / 5120$ from Figure 18. Table 2 shows these results with a least squares fit to the convergence rate $\kappa \approx 1.08$. To put this result in context, convergence rates for smooth flows using overlapping grids have previously been demonstrated to be $\kappa=O\left(\Delta x^{2}\right) \quad[17,16]$. However, for flows with solutions dominated by shocks a rate of $\kappa=O(\Delta x)$ is expected and for contact surface dominated flows a rate of $\kappa=O\left(\Delta x^{2 / 3}\right)$, for a second order method, is expected [28]. An extensive set of convergence results that demonstrate the capabilities of the FCT algorithm employed in this study can be found in [18]. Finally it should be noted that because simulations are run at fixed CFL, spatial refinement and temporal refinement are carried out in a coordinated manner. For explicit algorithms this is the usual refinement 



Figure 18: (Color) Shown here are the radiation pulses (left) and total radiated power (right) obtained using the linear current model $I=\sqrt{12} t$ on a pinch with $\rho_{\text {pre-fill }}=1 \times 10^{-3}$ and a stagnation point at $r=0.1$. The simulation is carried out in the $r-z$ plane and the resolutions, from coarse to fine, are represented by green, cyan, blue, black, red and maroon.

Table 1: Total radiated power, peak power, and time to peak power for the radiation results presented in Figure 18. Here $N$ is a measure of grid resolution with $h=1 / N$.

\begin{tabular}{cccc}
\hline \hline$N$ & total radiated power & peak power & time to peak power \\
\hline 160 & 2.261 & 118.7 & .9299 \\
\hline 320 & 1.833 & 172.0 & .9267 \\
\hline 640 & 1.528 & 215.3 & .9264 \\
\hline 1280 & 1.311 & 174.0 & .9260 \\
\hline 2560 & 1.203 & 196.8 & .9261 \\
\hline 5120 & 1.158 & 227.8 & .9257 \\
\hline \hline
\end{tabular}

procedure as refinement results for fixed $\Delta t$ or fixed $\Delta x$ can be difficult to interpret. For example temporal refinement at fixed $\Delta x$ will generally lead to divergent approximations [28].

The second radiation result demonstrates stagnation with the very difficult pre-fill density set at $\rho_{\text {pre-fill }}=$ $1 \times 10^{-6}$, the exterior density $\rho_{\text {exterior }}=5 \times 10^{-7}$ and stagnation on the center line. Here the current drive from (25) with $q=6$ and $I=0$ for $t \geq 1$ is used rather than the linear drive. The softening of the problem as a result of the $q=6$ current drive is more than made up for in the increased difficulty of resolving the stagnation on axis which makes numerical convergence extremely difficult. Simulations have been carried out for $h=1 / 160, h=1 / 320,1 / 640,1 / 1280,1 / 2560$ and $1 / 5120$. The reason for making $h=1 / 5120$ the finest resolution actually has nothing to do with computational cost, but rather a consideration of the artificial cutoff for the radius. In these simulations the minimum effective radius is chosen to be $1 \mathrm{e}-4$ and in order that no discretization points, except the one at the origin, fall within this radius the finest attainable resolution must have $h>1 / 10000$. To be consistent with previous resolution studies, $h=1 / 5120$ is a reasonable choice for the finest resolution. Figure 19 presents the radiation output and total radiated power as a function of time for these results and indicates strongly that convergence is near at hand for the finest resolution $h=1 / 5120$, but shows how difficult this problem is in terms of sufficient resolution for convergence. Indeed some aspects of the simulation seem to be well resolved such as the peak output location as a function of time, but even at this fine resolution the peak power is clearly not well resolved.

As before convergence is measured in two ways. The first takes the extrapolated convergence rate for the total radiated power at $t=1.0$ in Table 3 as in [27]. The result here is $\kappa \approx 0.37$ which is obviously not excellent, but still convergent and for such a difficult problem still considered reasonable. The second 
Table 2: Self convergence results for the $L_{1}$ norm of the radiation pulses from Figure 18 as compared to the finest resolution $h=1 / 5120$. Here $\kappa \approx 1.08$ is a least squares fit for the convergence rate and $N$ is a measure of grid resolution with $h=1 / N$.

\begin{tabular}{cc}
\hline \hline$N$ & self convergence error \\
\hline 160 & 2.614 \\
\hline 320 & 1.149 \\
\hline 640 & 0.5340 \\
\hline 1280 & 0.2672 \\
\hline 2560 & 0.1275 \\
\hline & \\
$\kappa$ & 1.08 \\
\hline \hline
\end{tabular}
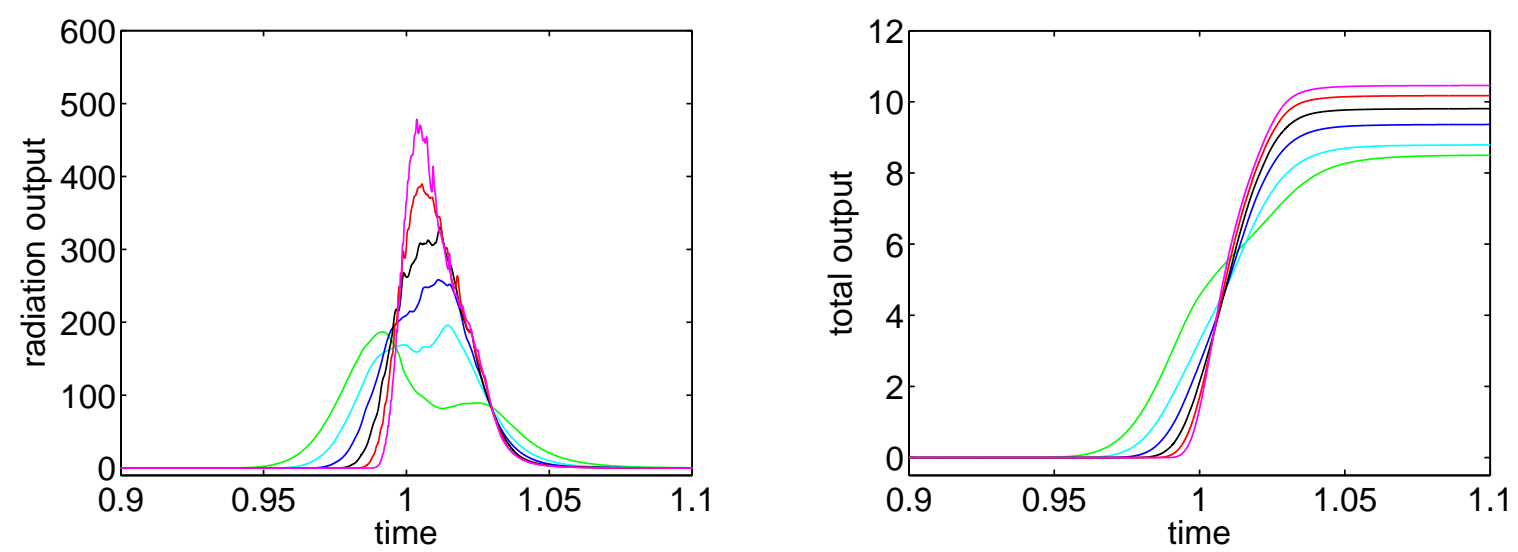

Figure 19: (Color) Shown here are the radiation pulses obtained using the current model (25) with $q=6$. The simulation is carried out in $(r, z)$ space with $r \in[0,1.25]$. The radiation output and total radiated power is plotted as a function of time with the simulations from coarse to fine represented by green, cyan, blue, black, red and maroon.

measure judges self convergence of individual radiation profiles in Figure 19 using the $L_{1}$ norm. Table 4 shows these results and demonstrates that even here the convergence rate is $\kappa \approx 0.75$ which is quite good.

\section{Concluding Remarks}

In this paper a phenomenological model for a magnetic drive source term for the momentum and total energy equations has been described. This body force term is designed to produce a Z-pinch like implosion that can be used in the development and evaluation of shock-hydrodynamics algorithms intended to be used as a basis for a larger Z-pinch simulation capability. The model uses a $\mathbf{J} \times \mathbf{B}$ Lorentz force source term which has been motivated by a $0-\mathrm{D}$ analysis of a thin shell or liner implosion.

The magnetic drive source term with a power law radial compression, and the corresponding current drive, was then demonstrated in an Euler system solver based on an FCT algorithm for mapped overlapping grids. The results of these computations verified the thin shell analysis as a limiting case with the numerical results showing good agreement with the analytic solution. As was further demonstrated, the magnetic drive source term was also useful in producing more complex implosion dynamics which we believe will be very useful in the evaluation of existing and proposed shock-hydrodynamics algorithms and implementations for Z-pinch type systems. The benefit of this approach is that the evaluation of these numerical methods can be carried out for a reasonably realistic set of Z-pinch like magnetic implosions without the requirement that a 
Table 3: Total radiated power, peak power, and time to peak power for the radiation results presented in Figure 19. Here $N$ is a measure of grid resolution with $h=1 / N$.

\begin{tabular}{cccc}
\hline \hline$N$ & total radiated power & peak power & time to peak power \\
\hline 160 & 8.500 & 186.9 & 1.015 \\
\hline 320 & 8.790 & 195.9 & 1.011 \\
\hline 640 & 9.365 & 258.5 & 1.011 \\
\hline 1280 & 9.808 & 330.4 & 1.012 \\
\hline 2560 & 10.18 & 389.7 & 1.006 \\
\hline 5120 & 10.46 & 478.5 & 1.003 \\
\hline \hline
\end{tabular}

Table 4: Self convergence results for the $L_{1}$ norm of the radiation pulses from figure 18 as compared to the finest resolution $h=1 / 5120$. Here $\kappa \approx 0.75$ is a least squares fit for the convergence rate and $N$ is a measure of grid resolution with $h=1 / N$.

\begin{tabular}{cc}
\hline \hline$N$ & self convergence error \\
\hline 160 & 10.74 \\
\hline 320 & 6.920 \\
\hline 640 & 4.450 \\
\hline 1280 & 2.654 \\
\hline 2560 & 1.290 \\
\hline$\kappa$ & 0.75 \\
\hline \hline
\end{tabular}

corresponding electro-magnetics capability be included into the implementation.

Finally we believe that with the inclusion of various types of radiation models and conduction effects this approach can also be useful for evaluation of some aspects of multi-physics radiation-hydrodynamics capabilities that are being considered for use in the context of Z-pinch type simulations. In this context an example of a simple radiation emission model was presented as well as simple convergence studies for the maximum power output and self convergence of the radiated power output.

\section{Acknowledgments}

We would like to thank Chris Jennings, Heath Hanshaw, Tom Brunner, Ray Lemke, Curt Ober, and Tim Trucano for helpful comments and indication of literature related to more realistic Z-pinch like implosion conditions.

This work was supported by a DOE NNSA ASC ALG effort and the DOE Office of Science AMR program at Sandia National Laboratory. Sandia is a multiprogram laboratory operated by Sandia Corporation, a Lockheed Martin Company, for the United States Department of Energy's National Nuclear Security Administration under contract DE-AC04-94AL85000.

\section{References}

[1] M. A. Liberman, J. S. D. Groot, A. Toor, R. B. Spielman, Physics of high-density Z-pinch plasmas, Springer Verlag, New York, 1999, pp. 7-10,19-28,44-54,133-163,239-243.

[2] M. K. Matzen et. al., Pulsed-power-driven high energy density physics and inertial confinement fusion research, Physics of Plasmas 12 (2005) 055503. 
[3] W. A. Stygar, H. C. Ives, D. L. Fehl, M. E. Cuneo, M. G. Mazarakis, J. E. Bailey, G. R. Bennett, D. E. Bliss, G. A. Chandler, R. J. Leeper, M. K. Matzen, D. H. McDaniel, J. S. McGurn, J. L. McKenney, L. P. Mix, D. J. Muron, J. L. Porter, J. J. Ramirez, L. E. Ruggles, J. F. Seamen, W. W. Simpson, C. S. Speas, R. B. Spielman, K. W. Struve, J. A. Torres, R. A. Vesey, T. C. Wagoner, X-ray emission from $z$ pinches at $10^{7}$ A: Current scaling, gap closure, and shot-to-shot fluctuations, Phys. Rev. E 69 (4) (2004) 046403.

[4] M. K. Matzen, Z pinches as intense x-ray sources for high-energy density physics applications, Physics of Plasmas 4 (5) (1997) 1519-1527.

[5] National Research Council National Academies, Frontiers in high energy density physics: The X-games of contemporary science, Springer Verlag, National Academies Press, 2003, pp. 18-19,34-119.

[6] S. V. Lebedev et. al., Laboratory astrophysics and collimated stellar outflows: The production of radiatively cooled hypersonic plasma jets, The Astrophysical Journal 564 (2002) 113-119.

[7] T.A. Gardiner et. al., MHD models and laboratory experiments of jets, Astrophysics and Space Science 287 (2003) 69-74.

[8] A. H. Cerqueira, E. m. De Gouveia Dal Pino, MHD numerical simulations of proto-stellar jets, Space Science Reviews 107 (2003) 337-340.

[9] P. Bellan, Miniconference on astrophysical jets, Physics of Plasmas 12 (2005) 058301.

[10] Foster et. al., High-energy-density laboratory astrophysics studies of jets and bow shocks, The Astrophysical Journal 634L (2005) 77-80.

[11] C. J. Garasi, D. E. Bliss, T. A. Mehlhorn, B. V. Oliver, A. C. Robinson, G. S. Sarkisov, Multidimensional high energy density physics modeling and simulation of wire array Z-pinch physics, Physics of Plasmas 11 (5) (2003) 2729-2737.

[12] M. Haines, S. lebedev, J. Chittenden, F. Beg, S. Bland, A. Dangor, The past, present and future of Z pinches, Physics of Plasmas 7 (5) (2000) 1672-1680.

[13] R. W. Lemke et. al., Amplitude reduction of nonuniformities induced by magnetic Rayleigh-Taylor instabilities in z-pinch dynamic hohlraums, Physics of Plasmas 12 (2005) 012703.

[14] S. A. Slutz et. al., Integrated two-dimensional simulations of dynamic hohlraum driven inertial fusion capsule implosions, Physics of Plasmas 13 (2006) 102701.

[15] W. D. Henshaw, D. W. Schwendeman, An adaptive numerical scheme for high-speed reactive flow on overlapping grids, J. Comput. Phys. 191 (2) (2003) 420-447.

[16] J. W. Banks, D. W. Schwendeman, A. K. Kapila, W. D. Henshaw, A high-resolution Godunov method for multi-material flows on overlapping grids, J. Comput. Physics 223 (2007) 262-297.

[17] J. W. Banks, W. D. Henshaw, J. N. Shadid, An FCT method for high-speed flows on structured overlapping meshes part I: Algorithm description, Research Report 2008-0294J, Sandia National Laboratory (2008).

[18] J. W. Banks, W. D. Henshaw, J. N. Shadid, An evaluation of the FCT method for high-speed flows on structured overlapping grids, J. Comput. Phys. (submitted).

[19] J. P. Boris, D. L. Book, Flux-corrected transport. I: SHASTA, a fluid transport algorithm that works, J. Comput. Phys. 11 (1973) 38-69.

[20] J. P. Boris, D. L. Book, K. Hain, Flux-corrected transport II: Generalizations of the method, J. Comput. Phys. 18 (1975) 248-283.

[21] J. P. Boris, D. L. Book, Flux-corrected transport III: Minimal-error FCT algorithms, J. Comput. Phys. 20 (1976) 397-431. 
[22] D. Kuzmin, R. Löhner, S. Turek (Eds.), Flux-Corrected Transport, Springer, Berlin Heidelberg, 2005, pp. 29-78.

[23] S. T. Zalesak, Fully multidimensional flux-corrected transport algorithms for fluids, J. Comput. Phys. 31 (1979) 335-362.

[24] J. P. Chittenden, S. V. Lebedev, B. V. Oliver, E. P. Yu, M. E. Cuneo, Equilibrium flow structures and scaling of implosion trajectories in wire array Z pinches, Physics of Plasmas 11 (3) (2004) 1118-1127.

[25] J. Chittenden, S. Lebedev, S. Bland, F. Beg, M. Haines, One-, two-, and three-dimensional modeling of the different phases of wire array z-pinch evolution, Physics of Plasmas 8 (5) (2001) 2305-2314.

[26] E. P. Yu et. al., Three-dimensional effects in trailing mass in the wire-array z pinch, Phy. Plasmas 15 (056301).

[27] C. J. Roy, Grid convergence error analysis for mixed-order numerical schemes, AIAA Journal 41 (4) (2003) 595-604.

[28] J. W. Banks, T. Aslam, W. J. Rider, On sub-linear convergence for linearly degenerate waves in capturing schemes, J. Comput. Phys. 227 (14) (2008) 6985-7002. 